Journal of Animal and Veterinary Advances 11 (15): 2801-2803, 2012

ISSN: $1680-5593$

(C) Medwell Journals, 2012

\title{
Expression of ETS Transcription Factor Etv4 in Busulfan-Treated Mouse Testis and Epididymis
}

\author{
Guanyu Che, Yang Liu, Yixue Sun, Bin Guo and Xueming Zhang \\ Jilin Province Key Laboratory of Animal Embryo Engineering, \\ The Center for Animal Embryo Engineering of Jilin Province, \\ College of Animal Sciences and Veterinary Medicine, Jilin University, \\ 130062 Changchun, China
}

\begin{abstract}
The importance of ETS transcription factor Etv 5 on the self-renewal of Spermatogonial Stem Cells (SSCs) has been well documented recently. Previously, the researchers reported that its peer Etv4 remained at a low level during the normal sexual maturity in mouse. It's well known that the development of spermatogonia and the expression of the regulatory molecules could be changed during the regeneration after damage of the seminiferous epithelia. To test whether the expression of Etv 4 was also altered in male reproductiove system after damage, expressions of Etv4 in testis and epididymis were examined in Busulfan-Treated Mouse Model. Mouse testis and epididymis were collected on the 0th, 3rd, 5th, 8th, 10th and 18th days after busulfan injection, respectively. The effect of busulfan treatment was confirmed histologically. In testis, Etv 4 mRNA declined evidently on day 5 and 8 and decreased significantly on day $10(\mathrm{p}<0.05)$ while it remained a relatively higher level at all time points in epididymis and decreased significantly on day $8(\mathrm{p}<0.05)$. Collectively, Etv 4 might be involved in the regeneration of seminiferous epithelia and the maturation of sperm in epididymis.
\end{abstract}

Key words: Etv4, testis, epididymis, busulfan, mouse, China

\section{INTRODUCTION}

The self-renewal and differentiation of Spermatogonial Stem Cells (SSCs) is the basis in maintaining of the spermatogenesis throughout life (Nakagawa et al., 2010). Recent studies suggest that Etv5, one of members of ETS transcription factor family, performs an essential regulation role during SSCs development (Chen et al., 2005; Schlesser et al., 2008). Etv 5 subfamily has three members including Etv1, Etv 4 and Etv5. They share a conserved DNA domain which can bind to similar site containing GGAA/T center sequence and participate in variety of cell development, differentiation, growth and transformation processes (Oikawa and Yamada, 2003). Previously, researchers have briefly reported that the expressions of Etv 5 and Etv1 in normal mouse testis reached a peak level during sexual maturity then decreased and maintained at a certain level while Etv 4 remained at a very low level during sexual maturity process. However, whether the expression of Etv 4 were changed under detrimental circumstance (busulfan-treatment) and played possible roles in SSCs development is still unclear.
DNA alkylating agent busulfan was widely used to deplete endogenous germ cells and promote the migration of transplanted spermatogonia by disrupting the junctions between Sertoli cells (Brinster and Zimmermann, 1994; Bucci and Meistrich, 1987). In this study, researchers further investigated the expression of Etv 4 in mouse male reproductive system and discussed its possible function on the proliferation and differentiation of spermatogonia by using Busulfan-Treated Mouse Model.

\section{MATERIALS AND METHODS}

Animals and reagents: Male Kunming White outbred strain mice ( $7-8$ week old, weight 30-35 g) were provided by the Experimental Animal Center of Jilin University (Certification No. scxk-Ji-2007-0003). All experiment mice were performed in accordance with the principles and procedures of Animal Ethics Committee of Jilin University. Busulfan and Dimethyl Sulphoxide (DMSO) were purchased from Sigma Chemicals (St Louis MO USA). All reagents used for molecular biology were from Takara (Dalian, China).

Corresponding Author: Xueming Zhang, College of Animal Sciences and Veterinary Medicine, Jilin University, 5333 Xi-an Road, 130062 Changchun, China 
Busulfan treatment: Busulfan $\left(3 \mathrm{mg} \mathrm{mL}^{-1}\right)$ was dissolved into DMSO diluted with sterile deionized water $(1: 1)$ at room temperature. Eighteen mice were intraperitoneally injected with busulfan $\left(10 \mathrm{mg} \mathrm{kg}^{-1}\right)$. Mice were divided into 6 groups randomly and sacrificed on the 0 th, 3rd, 5th, 8 th, 10th and 18th days, respectively. Testis and epididymis were collected and then kept at $-196^{\circ} \mathrm{C}$ until use. Tissue samples were collected from 3 animals $(n=3)$ for each time point.

Haematoxylin-Eosin (HE) staining: Briefly, frozen sections ( $10 \mu \mathrm{m}$ thick) from part of collected testes were air dried and fixed in $4 \%$ polyformaldehyde in PBS for $30 \mathrm{~min}$. After rinsing in running water for $30 \mathrm{sec}$, nuclear stain was carried out with haematoxylin for 3-5 min followed rinsing in running water for $30 \mathrm{sec}$, $1 \%$ hydrochloric acid-ethanol for $1 \mathrm{~min}$, running water for $30 \mathrm{sec}, 1 \%$ ammonia for $5 \mathrm{sec}$ and water for $30 \mathrm{sec}$. Sections were stained with $0.5 \%$ Eosin for $2 \mathrm{~min}$, dehydrated with gradient ethanol, treated with xylene twice for $10 \mathrm{~min}$ each, mounted and coverslipped for microscopy.

RNA extraction: Total RNA from mouse testis, epididymis was extracted using the Trizol reagent and then treated with Rnase-free Dnase I to eliminate genomic DNA. RNA was then diluted into equal concentration with Rnase-free distilled water. Oligo (dT) 18 was used in reverse transcription reactions. The resultant cDNA was frozen at $-20^{\circ} \mathrm{C}$ until use.

RT-PCR: First strand cDNA was synthesized from 2 ng of total RNA from testis or epididymis. Reverse transcription was conducted with the 1 st Strand cDNA Synthesis kit. The resultant cDNA was frozen at $-20^{\circ} \mathrm{C}$ untiluse. Primers of Etv4 (F: 5'-CACTCCTTCACATCTCC TC-3', R: 5'-AGCATTTGTTGGGTCATC-3', 499 bp) and internal control $\beta$-actin (F: 5'-GATATCGCTGCGCT GGT CGTC-3',R:5'-ACGCAGCTCATTGTAGAAGGTGTGG-3', 276 bp) were synthesized by Shanghai Sangon Biological Engineering Technology and Services CO., Ltd. (Shanghai, China).

The PCR conditions were as follows: initial denaturation at $94^{\circ} \mathrm{C}$ for $5 \mathrm{~min}, 94^{\circ} \mathrm{C}$ denaturation for $30 \mathrm{sec}$, annealing $30 \mathrm{sec}$ (Etv 4 at $55^{\circ} \mathrm{C}, \beta$-actin at $60^{\circ} \mathrm{C}$ ), extension at $72^{\circ} \mathrm{C}$ for $1 \mathrm{~min}$ and amplification $(28$ cycles for Etv 4 and 20 cycles for $\beta$-actin). PCR products were preceded for electrophoresis with $1.5 \%$ agarose gel. The target fragments were recovered and connected with the pGEM-T vector for transformation, screening and sequencing.
Statistical analysis: PCR amplification products of Etv4, $\beta$-actin and DNA marker (DL-2000) were preceded for electrophoresis with 1.5\% agarose gel and photographed with UV Gel Imaging System. Grayscale analysis was carried out in triplicate with Gel-Pro Analyzer 4.0 software. The mean values of Etv $4 / \beta$-actin in same tissue on time point were used as the expression level of Etv4. Statistical analysis was performed by ANOVA using SPSS 13.0 and the difference was considered significant when $\mathrm{p}<0.05$.

\section{RESULTS}

Histological examination of the Busulfan-Treated Model: HE staining showed that morphological change occurred in the seminiferous tubules along time going after busulfan injection. Small vacuoles could be observed in the seminiferous epithelia on day 3 and the seminiferous epithelia became looser and more vacuolated on day 5,8 and 10 which suggest that the depletion of endogenous spermatogonia by busulfan was more and more serious. Afterwards, recovery occurred and the seminiferous epithelia on day 18 revealed no obvious difference from that of day 3 or even day 0 implying spermatogenic cells regenerated gradually.

Expression of Etv4 mRNA in testis and epididymis: The electrophoresis of the PCR products of Etv4 and $\beta$-actin in testis and epididymis was showed in Fig. 1. Etv4 mRNA band was weak on day 5,8 and especially day 10 in testis (Fig. 1a) while evidently weak on day 8 in epididymis (Fig. 1b). The semi-quantitative RT-PCR analys is was showed in Table 1. In testis, Etv4 mRNA decreased on day 5,8 and especially on day $10(\mathrm{p}<0.05)$, compared with that on day 0 . While in epididymis, its expression maintained higher levels on all time points compared with that in testis and significantly decreased on day $8(\mathrm{p}<0.05)$ compared with that on day 0 .

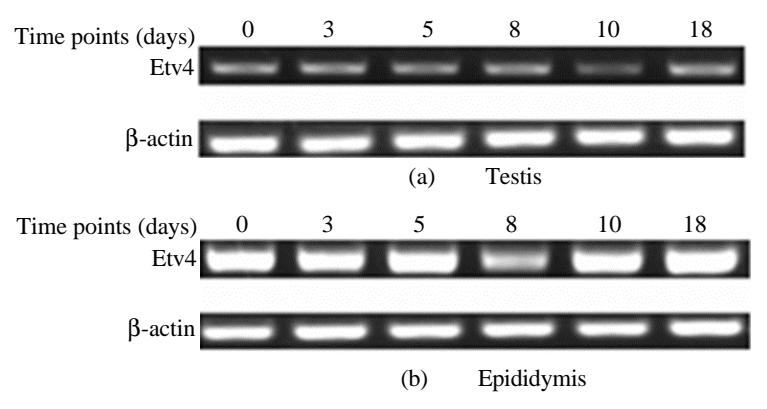

Fig. 1: RT-PCR analysis of Etv4 mRNA in busulfan-treated mouse testis and epididymis 
Table 1: Expressions of Etv4 mRNA in busulfan-treated mouse testis and epididymis

\begin{tabular}{|c|c|c|c|c|c|c|}
\hline \multirow[b]{2}{*}{ Tissue } & \multicolumn{6}{|c|}{ Days after busulfan treatment } \\
\hline & 0 & 3 & 5 & 8 & 10 & 18 \\
\hline Testis & $0.91 \pm 0.04$ & $0.91 \pm 0.06$ & $0.72 \pm 0.08$ & $0.76 \pm 0.11$ & $0.49 \pm 0.05^{*}$ & $0.93 \pm 0.06$ \\
\hline Epididymis & $3.26 \pm 0.15$ & $2.50 \pm 0.17$ & $2.97 \pm 0.15$ & $1.82 \pm 0.24^{*}$ & $2.85 \pm 0.19$ & $2.56 \pm 0.20$ \\
\hline
\end{tabular}

Data represents means \pm standard deviation $(n=3)$. * $p<0.05$ and $* * p<0.01$ compared with day 0

\section{DISCUSSION}

ETS transcription factor family has $>30$ members which are widely involved in cells development, differentiation, growth and transformation process and play important roles in regulating many physiological and pathological processes (Oikawa and Yamada, 2003; Lettice et al., 2012; Hollenhorst et al., 2011). The essential function of ETS factor Etv5 in the transcriptional regulation of SSCs niches has been demonstrated recently (Chen et al., 2005; Schlesser et al., 2008). In view of the homology in structure, similarity in function and wide distribution between Etv5 and its peers (Etv1 and Etv4), researchers briefly reported the expressions of Etv1 remaining at relatively high level but Etv 4 at very low level in normal testis. However, the expression pattern of them might be changed during regeneration after damage (busulfan treatment).

Busulfan is a DNA alkylating agent commonly used to eliminate male germ cells in reproductive biological research. Its required dosage reported in the literature varies widely between different species (Wang et al., 2010). In this study, researchers administrated busulfan at a submarginal dose to Establish Mouse Model. The morphological analysis indicated that the depletion and regeneration process of seminiferous epithelia was accordant with previous report (Nakagawa et al., 2010; Anjamrooz et al., 2007) which suggests that we produced the mouse model successfully. Similarly, the expression of Etv 4 in testis revealed a decline and then recovery which is possibly correlated with the changes of seminiferous epithelia. The higher level of Etv 4 in epididymis might imply that it is involved in the process of sperm maturation in epididymis.

\section{CONCLUSION}

The change of the expression pattern of Etv 4 mRNA in busulfan-treated mouse testis was correlated with the regeneration of the seminiferous epithelia after damage. The high level of Etv4 mRNA in epididymis might be involved in the maintenance of sperm maturation in detrimental environment.

\section{ACKNOWLEDGEMENTS}

This research was financially supported by the National Natural Science Foundation of China (30771555), the Functional Expenses of Basic Scientific Research of
Jilin University (200903333) and Science and Technology Development Project of Jilin Province, China (201105021).

\section{REFERENCES}

Anjamrooz, S.H., M. Movahedin, S.J. Mowla and S.P. Bairanvand, 2007. Assessment of morphological and functional changes in the mouse testis and epididymal sperms following busulfan treatment. Iran Biomed. J., 11: 15-22.

Brinster, R.L. and J.W. Zimmermann, 1994. Spermatogenesis following male germ-cell transplantation. Proc. Natl. Acad. Sci. USA., 91: 11298-11302.

Bucci, L.R. and M.L. Meistrich, 1987. Effects of busulfan on murine spermatogenesis: cytotoxicity, sterility, sperm abnormalities and dominant lethal mutations. Mutat. Res., 176: 259-268.

Chen, C., W. Ouyang, V. Grigura, Q. Zhou and $\mathrm{K}$. Karnes et al., 2005. ERM is required for transcriptional control of the spermatogonial stem cell niche. Nature, 436: 1030-1034.

Hollenhorst, P.C., L. Paul, M.W. Ferris and B.J. Graves, 2011. The ETS gene ETV4 is required for anchorageindependent growth and a cell proliferation gene expression program in PC3 prostate cells. Genes Cancer, 1: 1044-1052.

Lettice, L.A., I. Williamson, J.H. Wiltshire, S. Peluso and P.S. Devenney et al., 2012. Opposing functions of the ETS factor family define Shh spatial expression in limb buds and underlie polydactyly. Dev. Cell., 22: 459-467.

Nakagawa, T., M. Sharma, Y. Nabeshima, R.E. Braun and S. Yoshida, 2010. Functional hierarchy and reversibility within the murine spermatogenic stem cell compartment. Science, 328: 62-67.

Oikawa, T. and T. Yamada, 2003. Molecular biology of the Ets family of transcription factors. Genetics, 303: 11-34.

Schlesser, H.N., L. Simon, M.C. Hofmann, K.M. Murphy, T. Murphy, R.A. Hess and P.S. Cooke, 2008. Effects of ETV5 (ETS variant gene 5) on testis and body growth, time course of spermatogonial stem cell loss and fertility in mice. Biol. Reprod., 78: 483-489.

Wang, D.Z., X.H. Zhou, Y.L. Yuan and X.M. Zheng, 2010. Optimal dose of busulfan for depleting testicular germ cells of recipient mice before spermatogonial transplantation. Asian J. Androl., 12: 263-270. 